\title{
On an Infinite-Dimensional Group
}

\author{
Moshé Flato and Daniel Sternheimer \\ Physique Mathématique, Collège de France, Paris
}

Received March 17, 1969

\begin{abstract}
We present here an infinite-dimensional Lie algebra, semi-direct product of the Poincare Lie algebra $\mathscr{P}$ by an infinite-dimensional abelian Lie algebra. It gives rise to Schur-irreducible subgroups of the unitary group of the (separable) Hilbert space, with a discrete mass-spectrum (real positive isolated mass-eigenvalues). Some related mathematical problems are also examined.
\end{abstract}

\section{Introduction}

In [1] an example was given of a Poincaré partially-integrable local representation of a fifteen-dimensional Lie algebra, giving rise to a discrete mass-spectrum. Though this example is physical, we cannot get a unitary representation of a Lie group (a thing which would have been of technical commodity) out of it, due to the lack of common analytic vectors [2]. In particular, due to the generator $q$, we are forced in [1] to introduce two domains $S_{0}$ and $S_{\pi}$, the former as a dense domain on which all the commutation relations of the 15-dimensional Lie algebra are verified, the latter being the mass-spectrum domain - on which a 14-dimensional subalgebra is integrable, the trouble being caused by $q$ which even does not leave this domain $S_{\pi}$ invariant.

As was already hinted in [1], and because of the results of [3], this example is an optimal one (and of physical interest) within the framework of finite-dimensional Lie algebras containing the Poincaré Lie algebra. Of course the necessity of introducing $S_{0}$ besides $S_{\pi}$, as well as the fact that we have only partial integrability of the representation are caused by the fact that $q$ is not a periodic function. The last suggests ${ }^{1}$ to replace multiplication by $q$ by multiplication by $\sin q$ and $\cos q$ (supposing for simplicity $a=2 \pi$ in the notations of [1]). Doing so we get an infinite-dimensional Lie algebra with a common dense set of analytic vectors, namely this Lie algebra is integrable to a group representation. This last fact shows us that we have essentially only two types of possibilities of overcoming the negative results of [3]: either we have mass-

${ }^{1}$ This suggestion was made to us by E. P. Wigner in Trieste in June 1968, and was at the origin of this work. 
spectrum in a finite-dimensional Lie algebra with only partial integrability to (e.g.) Poincaré, or else we have mass-spectrum in an integrable representation of a Lie algebra containing Poincaré, in which case the Lie algebra must be infinite-dimensional.

Indeed in what follows we shall study mathematically an "infinitedimensional version" of [1]. We shall see that in this case the translational ideal of $\mathscr{P}$ is even not nilpotent in the entire infinite-dimensional Lie algebra (its nilpotency was a basic point in the proofs of the negative results of [3]). Before we pass to the example itself, we shall say a few words about its possible physical interpretation. Our example will contain a kind of tensor product of polynomials (or other nicely behaved functions) in the energy-momentum generators, which will play the role of external observables of the system of particles, by a representation of the two-dimensional Euclidean group, generated by $\sin q, \cos q$, and $\partial_{q}$.

In this hypothetical model the two-dimensional Euclidean group plays the role of an internal symmetry: we have rotations around a third axis in isotopic-space, as well as real translations in the (1-2) plane. $\sin q, \cos q$ and $\partial_{q}$ are internal observables of the theory.

Of course one understands immediately that such an example is generalizable with any (more realistic) internal symmetry, and that the utilization of (e.g.) $S U(3)$ in such a context can give quite richier applications. But now let us pass to the example itself.

\section{The Infinite-Dimensional Lie Algebra}

As in [1], we shall start with the infinitesimal skew-adjoint generators $\left(M_{\mu \nu}^{\prime}, P_{\mu}^{\prime}\right)$ of (in order to fix ideas and keep in view physical applications) an irreducible unitary representation $D_{j}^{+}\left(m_{0}\right)$ of the Poincaré group on a separable Hilbert space $H_{1}$. On the (complete) Hilbert tensor product $H=L^{2}(0,2 \pi) \hat{\otimes} H_{1}$ we shall then consider the skew-adjoint operators $M_{\mu \nu}=I \bar{\otimes} M_{\mu \nu}^{\prime}, P_{\mu}=I \bar{\otimes} P_{\mu}^{\prime}$ (where $I$ stands for the identity operator on $L^{2}(0,2 \pi)$ and the bar for closure), and $P_{\mu}\left(i \partial_{q}\right)$ which together with $M_{\mu \nu}$ generate a Lie algebra $D(\mathscr{P})$ representing the Poincare Lie algebra on $H$ - here also, $i \partial_{q}$ is taken self-adjoint on the domain of absolutely continuous functions in $q \in(0,2 \pi)$ with the periodic boundary condition in $q$. All operators are essentially skew-adjoint on a common domain $S_{\pi}$ consisting of $C^{\infty}$ functions periodic in $q$ (with rapid decrease in the momentum-variables $\boldsymbol{p}$ if $H_{1}$ is realized, as usual, as a Hilbert space of functions on a hyperboloid), and $D(\mathscr{P})$ gives rise to a unitary representation $D(\tilde{\boldsymbol{P}})$ of the (universal covering $\tilde{\boldsymbol{P}}$ of the) Poincare group, decomposable (cf. [1]) into $\sum_{n=1}^{\infty} D_{j}^{+}\left(n m_{0}\right) \oplus D_{j}(0) \oplus \sum_{n=1}^{\infty} D_{j}^{-}\left(n m_{0}\right)$. 
Now (following Wigner's suggestion) we add to $D(\mathscr{P})$ the fourdimensional abelian algebra $D\left(A_{1}\right)$ generated by the (skew-adjoint) operators $P_{\mu} \sin q$. The latter is invariant by commutation with the $M_{\mu \nu}$ and gives the ten-dimensional abelian algebra $D\left(A_{2}\right)$, generated by the $P_{\mu} P_{v} i \cos q$, by commutation with the $P_{v}\left(i \partial_{q}\right)$. On $D\left(A_{2}\right)$, the $M_{\mu v}$ act through the tensor product of two representations $D(1 / 2,1 / 2)$ of the Lorentz Lie algebra $\mathscr{L}$.

Continuing this process we shall get the $\left(\begin{array}{c}n+3 \\ 3\end{array}\right)$ abelian algebra $D\left(A_{n}\right)$ generated by the $n$-products of the $P_{\mu}$ 's multiplied by $\sin q$ or $i \cos q$, according to the parity (odd or even) of $n$, which are all essentially skew-adjoint on the domain $S_{\pi}$. The $M_{\mu \nu}$ act on $D\left(A_{n}\right)$ through the $n$th tensorial power of $D(1 / 2,1 / 2)$ and leave it invariant, while the $P_{\mu}\left(i \partial_{q}\right)$ transform it into $D\left(A_{n+1}\right)$ - which shows they are not nilpotent (for the adjoint representation).

The commutation relations thus defined give us the infinite-dimensional Lie algebra $G$, semi-direct product $\mathscr{P} \cdot A_{0}$ of $\mathscr{P}$ by the infinitedimensional abelian Lie algebra $A_{0}=\sum_{n=1}^{\infty} A_{n}$, the sum being considered for the moment as an algebraic direct sum of Lie algebras. Moreover all operators in $D(G)$ are essentially skew-adjoint on a dense subspace of $H$, contained in $S_{\pi}$ and invariant under $D(G)$. Thus we can define not only the unitary group $D(\tilde{\boldsymbol{P}})$ representing $\tilde{\boldsymbol{P}}$ but also abelian unitary groups $D\left(\boldsymbol{B}_{n}\right)$ corresponding to $D\left(A_{n}\right)$ and representing the vectorgroup of translations in $\left(\begin{array}{c}n+3 \\ 3\end{array}\right)$-dimensional space, and therefore also an abelian group $D\left(\boldsymbol{B}_{0}\right)$ corresponding to $D\left(A_{0}\right)$ - and even a group of unitary operators in $H$, algebraically generated by $D(\tilde{\boldsymbol{P}})$ and $D\left(\boldsymbol{B}_{0}\right)$.

Now if $f_{1}(p)$ [resp. $f_{2}(p)$ ] is any odd [resp. even] entire function of exponential type in $p$ we can define on a subspace of $S_{\pi}$ of functions sufficiently rapidly decreasing (in $p$ ). the (essentially skew-adjoint) operator $f_{1}(P) \sin q+i f_{2}(P) \cos q$. Such operators will still have in common with the formerly defined operators in $D(G)$ a dense set of analytic vectors (for instance entire functions in $\boldsymbol{p}$ decreasing on the real 3-space like $\exp \left(-\exp |\boldsymbol{p}|^{2}\right)$, times trigonometric polynomials in $\left.q\right)$. This remark will be useful in the next paragraph.

Moreover, here also, the Lie algebra $D^{\prime}(G)$ is a Schur-irreducible set of operators in $H$ [any bounded operator commuting to it - on a dense subspace e.g. (cf. [1]) - is a multiple of identity], since the Lie algebra generated by $\left(\partial_{q}, \sin q, \cos q\right)$ is Schur-irreducible in $L^{2}(0,2 \pi)$. The latter gives even rise to a (Schur, and topologically) irreducible (nonunitary) representation of the Euclidean group of a two-dimensional 
space (e.g. by exponentiating the generators restricted to a dense domain of common analytic vectors, and then by taking the closure of the obtained bounded operators).

\section{Completions of the Lie Algebra, and Semi-Direct Product Structure of Corresponding Groups}

1. Let $L$ be the free Lie algebra generated over two elements $x$ and $y$ [4]. Its enveloping algebra is the tensor algebra $T$ over the linear space generated by $x$ and $y$. Denote by $T^{*}$ the completion of $T$ with respect to its natural graduation, i.e. the associative algebra of formal (noncommutative) power series in $x$ and $y$, and by $L^{*}$ the closure of $L$ in $T^{*}$.

It is known that $\log (\operatorname{Exp}(x) \operatorname{Exp}(y) \operatorname{Exp}(-x)) \in L^{*}$ and is equal to $\operatorname{Ad}(\operatorname{Exp}(x)) \cdot y$, which in turn is equal to $\operatorname{Exp}(\operatorname{ad} x) \cdot y$. This can be easily seen: $\operatorname{ad} x \cdot y=[x, y]=x y-y x=\left(L_{x}-R_{x}\right) y$ in $T$ (and $T^{*}$ ) and $L_{x}$ and $R_{x}$ (left and right multiplication by $x$ ) commute; thus

$$
\operatorname{Exp}(\operatorname{ad} x)=\sum_{0}^{\infty} \frac{1}{n !}\left(L_{x}-R_{x}\right)^{n}=\left(\sum_{j=0}^{\infty} \frac{1}{j !} L_{x}\right)\left(\sum_{k=0}^{\infty} \frac{1}{k !}\left(-R_{x}\right)^{k}\right)=\operatorname{Ad}(\operatorname{Exp} x) ;
$$

and evidently

$$
\operatorname{Exp}(\operatorname{Ad}(\operatorname{Exp} x) y)=\operatorname{Exp}(\operatorname{Exp}(x) y \operatorname{Exp}(-x))=\operatorname{Exp}(x) \operatorname{Exp}(y) \operatorname{Exp}(-x) \text {. }
$$

2. We shall now apply these remarks to our case. We have

$$
\begin{aligned}
\operatorname{Ad}\left(\exp \left(t_{\mu} P_{\mu}\left(i \partial_{q}\right)\right)\right) \sin q & =\exp \left(\operatorname{ad}\left(t_{\mu} P_{\mu}\left(i \partial_{q}\right)\right)\right) \sin q \\
& =\cosh \left(t_{\mu} P_{\mu}\right) \sin q+i \sinh \left(t_{\mu} P_{\mu}\right) \cos q
\end{aligned}
$$

and similarly

$$
\operatorname{Ad}\left(\exp \left(t_{\mu} P_{\mu}\left(i \partial_{q}\right)\right)\right) i \cos q=\sinh \left(t_{\mu} P_{\mu}\right) \sin q+i \cosh \left(t_{\mu} P_{\mu}\right) \cos q .
$$

Now let $F_{1}$ (resp. $F_{2}$ ) be the linear space of odd (resp. even) entire functions of exponential type in the four variables $p_{\mu}$ which are linear combinations of products of a polynomial in the $p_{\mu}$ by finite products of $\sinh \left(t_{\mu} p_{\mu}\right)$ and $\cosh \left(t_{\mu}^{\prime} p_{\mu}\right)$, where $t=\left(t_{\mu}\right), t^{\prime}=\left(t_{\mu}^{\prime}\right) \in R^{4}$ and may be different from one term to another. Then it is easy to check that $\operatorname{Ad}\left(\exp \left(t_{\mu \nu} M_{\mu \nu}\right)\right)$, with $\left(t_{\mu \nu}\right) \in \boldsymbol{R}^{6}$ and summation for $\mu<v$, and therefore also $\operatorname{Ad}(D(\tilde{\boldsymbol{P}}))$, leave invariant the abelian Lie algebra $D\left(\tilde{A_{0}}\right)$ generated by the operators $f_{1}(P) \sin q+i f_{2}(P) \cos q$ when $f_{1} \in F_{1}, f_{2} \in F_{2}$, and $P=\left(P_{\mu}\right)$.

From what we have seen, there is an abelian unitary group $D\left(\tilde{\boldsymbol{B}}_{0}\right)$ corresponding to $D\left(\tilde{A_{0}}\right)$, and which can be defined by exponentiation from it on a dense set of common (to $D(\tilde{\boldsymbol{P}})$ also) analytic vectors. Denote by $D(\tilde{G})$ the semi-direct product $D(\mathscr{P}) \cdot D\left(\tilde{A_{0}}\right)$, where $D(\mathscr{P})$ acts as usual on 
$D\left(\tilde{A_{0}}\right)$ by ad $P_{\mu}\left(i \partial_{q}\right)$ and ad $M_{\mu v}$. To this Lie algebra we are now in position to associate the group $\tilde{\mathfrak{b}}_{D}=D(\tilde{\boldsymbol{P}}) \cdot D\left(\tilde{\boldsymbol{B}}_{0}\right)$, since $D\left(\tilde{\boldsymbol{B}}_{0}\right)$ is invariant under the automorphisms $\operatorname{Ad} D(\tilde{\boldsymbol{P}})$ of the unitary group of the Banach algebra $\mathscr{L}(H)$ of bounded operators on $H$ [remember that any element of $\tilde{\boldsymbol{P}}$ is a (finite) product of exponentials].

3. The group $\mathfrak{G}_{D}$ has a semi-direct product structure [which was not the case of the group algebraically generated by $D(\tilde{\boldsymbol{P}})$ and the $\left.D\left(\boldsymbol{B}_{n}\right)\right]$. Moreover, it is a topological semi-direct product, in the sense that the representation $\tilde{\boldsymbol{P}} \rightarrow \operatorname{Ad}(D(\tilde{\boldsymbol{P}}))$ of $\tilde{\boldsymbol{P}}$ into the group of automorphisms of $D\left(\tilde{\boldsymbol{B}}_{0}\right)$ is continuous with respect to the "strong-strong" topology defined by the following semi-norms: $a \mapsto\|a(b) \varphi\|$ (norm in $H$, with any $b \in D\left(\tilde{\boldsymbol{B}}_{0}\right)$ and $\varphi \in H)$. Indeed one sees easily that, for any $f_{1}(P) \sin q+i f_{2}(P) \cos q$ in $D\left(\tilde{A}_{0}\right)$ and $\varphi \in H$, the function

$$
\begin{aligned}
\left(t_{\mu}\right) \mapsto \exp \left[\left(f_{1}(P) \cosh \left(t_{\mu} P_{\mu}\right)+f_{2}(P) \sinh \left(t_{\mu} P_{\mu}\right)\right) \sin q\right. \\
\left.+i\left(f_{1}(P) \sinh \left(t_{\mu} P_{\mu}\right)+f_{2}(P) \cosh \left(t_{\mu} P_{\mu}\right)\right) \cos q\right] \varphi
\end{aligned}
$$

is a continuous function $R^{4} \rightarrow H$; and similarly for the Lorentz part, which is generated by the $\exp \left(t_{\mu \nu} M_{\mu v}\right)$, since we have

$$
\begin{aligned}
\operatorname{Ad} \exp \left(t_{\mu \nu} M_{\mu \nu}\right) \cdot \exp \left(f_{1}(P) \sin q\right. & \left.+i f_{2}(P) \cos q\right) \\
& =\exp \left[\tilde{f}_{1}\left(t_{\mu \nu} ; P\right) \sin q+i \tilde{f}_{2}\left(t_{\mu \nu} ; P\right) \cos q\right]
\end{aligned}
$$

where $\tilde{f_{\alpha}}$ belongs to $F_{\alpha}(\alpha=1,2)$ with respect to $P$, is a polynomial whenever $f_{\alpha}$ is a polynomial (the degree of $\tilde{f}_{\alpha}$ being not greater than that of $f_{\alpha}$ ) and is an entire function of exponential type in $\left(t_{\mu v}\right) \in R^{6}$ (bounded with respect to the parameters associated with a compact subgroup). The $D\left(\boldsymbol{B}_{n}\right)$ are invariant under the Lorentz part (but not under the translations).

4. The group $D\left(\tilde{\boldsymbol{B}}_{0}\right)$ is a normed group, but not a Banach Lie group (respectively to this norm) since it is not complete in the norm topology of $\mathscr{L}(H)$. We shall thus consider also the unitary group $D\left(\hat{\boldsymbol{B}}_{0}\right)$ of the commutative $C^{*}$-algebra generated by the $D\left(\boldsymbol{B}_{n}\right)$. It contains obviously $D\left(\tilde{\boldsymbol{B}}_{0}\right)$, is a Banach Lie group, and we can define in a similar way the topological semi-direct product $\hat{\boldsymbol{G}}_{D}=D(\tilde{\boldsymbol{P}}) \cdot D\left(\hat{\boldsymbol{B}}_{0}\right)$, which is a kind of completion of $\mathfrak{G}_{D}$. The (unitary abelian) normal subgroups $D\left(\tilde{\boldsymbol{B}}_{0}\right)$ and $D\left(\hat{\boldsymbol{B}}_{0}\right)$ of (respectively) $\mathfrak{G}_{D}$ and $\hat{\mathfrak{G}}_{D}$ can be interpreted as abelian gauges, related to Poincaré by a semi-direct structure.

\section{Concluding Remarks}

1. An interesting question raised by our treatment is to get an intrinsic definition of a Banach (or normed) Lie group corresponding to some completion of the abelian Lie algebra $A_{0}$. The definition we gave 
of $D\left(\hat{\boldsymbol{B}}_{0}\right)$ and $\hat{\mathfrak{G}}_{D}$ depends a priori on the considered representation. Abstract definitions of $C^{*}$-algebras do exist, which suggests that at least for some classes of representations of $\tilde{\boldsymbol{P}}$ (from which we start) abstract definitions of $D\left(\hat{\boldsymbol{B}}_{0}\right)$ and of $\hat{\mathfrak{G}}_{D}$ will be possible. But in any case $D\left(\hat{\boldsymbol{B}}_{0}\right)$ is very big, though it can be made even bigger by completion to the unitary group $D\left(\boldsymbol{B}_{0}^{*}\right)$ of the abelian von Neumann algebra it generates, and it would be of interest to define abstractly "analytic" (in the sense of $D\left(\tilde{\boldsymbol{B}}_{0}\right)$ ) subgroups of it.

Concerning $\tilde{\mathfrak{G}}_{D}$ and $D\left(\tilde{\boldsymbol{B}}_{0}\right)$, an intrinsic definition of abstract groups $\tilde{G}=\tilde{\boldsymbol{P}} \cdot \tilde{\boldsymbol{B}}_{0}$ and $\tilde{\boldsymbol{B}}_{0}$ (respectively), of which the former are representations, can be easily given by means of the construction of formal Lie groups. Indeed $\tilde{\boldsymbol{B}}_{0}$ is the abelian group defined as exponentials of elements of the abelian Lie algebra $\tilde{A}_{0}$, the elements of which are of the form $S f_{1}(T)+i C f_{2}(T)$, with $f_{\alpha} \in F_{\alpha}(\alpha=1,2), T$ being a generic element of the translational ideal $\mathscr{T}_{a}$ of an abstract Poincaré algebra [so that $f_{\alpha}(T)$

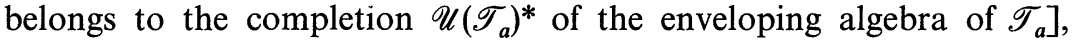
and where $S$ and $C$ are two additional generators commuting between themselves and with $\mathscr{T}_{a}$. We thus define, exactly as before, the abstract Lie algebra $\tilde{G}$ as the semi-direct product $\mathscr{P} \cdot \tilde{A}_{0}$, and the abstract (uniquely defined) corresponding group $\left(\mathfrak{F}\right.$ as the semi-direct product $\tilde{\boldsymbol{P}} \cdot \tilde{\boldsymbol{B}}_{0}$.

The insertion of $D\left(\tilde{\boldsymbol{B}}_{0}\right)$ between $D\left(\boldsymbol{B}_{0}\right)$ and $D\left(\hat{\boldsymbol{B}}_{0}\right)\left(C D\left(\boldsymbol{B}_{0}^{*}\right)\right)$ reminds us of the insertion of the henselization [5] $\tilde{\mathscr{R}}$ (algebraic functions) of the local ring $\mathscr{R}$ of (e.g.) an affine space between $\mathscr{R}$ (polynomials) and its "analytic closure" $\overline{\mathscr{R}}$ (analytic functions), contained in its completion $\hat{\mathscr{R}}$ (formal power series). $D\left(\tilde{\boldsymbol{B}}_{0}\right)$ is a kind of "exponential closure" of $D\left(\boldsymbol{B}_{0}\right)$ in its topological completion $D\left(\hat{\boldsymbol{B}}_{0}\right)$. This suggests that algebraic methods could be of use in the interesting problem of well defining intermediate ("analytic") subalgebras $\tilde{\mathscr{A}}$ between a $C^{*}$-algebra $\hat{\mathscr{A}}$ and a (sufficiently "small") subalgebra $\mathscr{A}$ generating $\hat{\mathscr{A}}$, so that in our case $D\left(\tilde{\boldsymbol{B}}_{0}\right)$ would be the unitary group of such an intermediate subalgebra.

2. The method of construction of $\mathfrak{G}_{D}$ gives us all representations of the group $\mathbf{5}$ that can be obtained from representations of $\tilde{\boldsymbol{P}}$ by "tensorproduct" of the translation generators with the Lie algebra $\left\{\partial_{q} ; \sin q\right.$, $\cos q\}$. It remains an open question whether other integrable skew-adjoint representations of the Lie algebra $\tilde{G}$ can be found. [Notice that in all our representations we have the relation $\left.S^{2}+C^{2}=1\right]$.

3. We know that $\mathscr{P}$ is "almost maximal" in the $\mathfrak{s u}(2,2)$ Lie algebra. More exactly, the inhomogeneous Lorentz Lie algebra, plus dilatations of space-time (the latter being a one-dimensional algebra) is maximal in the conformal Lie algebra. Moreover, the Euclidean Lie algebra (of a 2-dimensional space) is a maximal parabolic subalgebra of $\mathfrak{s}(2, \boldsymbol{C})$. Both maximalities can be easily seen with graduations and filtrations: if we add to the generators $m_{\mu \nu}$ and $p_{\mu}$ of $\mathscr{P}$ the five generators $c_{\mu}, d$ 
with $\left\{m_{\mu v}, c_{\mu}\right\} \approx \mathscr{P},\left[d, c_{\mu}\right]=-c_{\mu},\left[d, p_{\mu}\right]=p_{\mu},\left[p_{\mu}, c_{v}\right]=2\left(g_{\mu v} d+m_{\mu v}\right)$, we get the graduation $\mathfrak{s u}(2,2)=L^{-1}+L^{0}+L^{1}$, where $L^{-1}=\left\{c_{\mu}\right\}$, $L^{0}=\left\{m_{\mu \nu}, d\right\}$ and $L^{1}=\left\{p_{\mu}\right\}$. We can also write $\mathscr{L}=L_{-1} \supset L_{0} \supset L_{1}$, where $L_{1}$ is (e.g.) the abelian subalgebra with nilpotent elements $\left\{m_{12}+m_{02}, m_{13}+m_{03}\right\}$ and $L_{0}$ is generated by $L_{1}$ and the Cartan subalgebra $\left\{m_{23}, m_{01}\right\}$. The subalgebra generated by $L_{1}$ and $m_{23}$ of the (maximal parabolic) subalgebra $L_{0}$ is isomorphic to the real Lie algebra $\left\{\partial_{q} ; \sin q, \cos q\right\}$.

Our Lie algebra $G$ can be considered in many ways as a filtered Lie algebra. It seems that the most reasonable one, in view of the preceding decompositions, is the following:

$$
\begin{aligned}
& D(G)=L_{0}^{\prime}=\left\{M_{\mu v} ; L_{1}^{\prime}\right\} \supset L_{1}^{\prime}=\left\{P_{\mu}\left(i \partial_{q}\right), P_{\mu} \sin q ; L_{2}^{\prime}\right\} \supset L_{2}^{\prime} \\
&=\left\{i P_{\mu} P_{\nu} \cos q ; L_{3}^{\prime}\right\} \supset \cdots .
\end{aligned}
$$

The natural question is then how to "complete" our filtered Lie algebra $G$ so that to obtain one of the "simple" algebras classified by Cartan (for a bibliography on primitive filtered Lie algebras and definitions concerned with this subject, we refer to [6]) - here we must, at least in the beginning, forget the operator origin of $D(G)$ to concentrate on its structure. $G$ being some kind of "tensor product" of $\mathscr{P}$ (more precisely, of $L^{1}$ ) by $\left\{L_{1}, m_{23}\right\}$, it is possible that the completion of these to primitive algebras will give a hint at the structure of the wanted infinite-dimensional primitive-Lie algebra. Conversely, once the latter is found, the representations of $G$ we constructed here will give a hint at the way how to get some of the linear representations of this primitive algebra (a thing which has not been done yet in the mathematical literature, and of great interest).

4. Most of what has been said here for $\tilde{\boldsymbol{P}}$ can be generalized to semidirect products of a semi-simple Lie group by an abelian normal subgroup defined by an irreducible representation (what we call "special affine groups"). The unitarity of the representations can also be dropped without major difficulties, and other examples of filtered (or graded) infinite-dimensional Lie algebras of operators can be constructed along similar lines.

Acknowledgement. The authors wish to thank Eugene Wigner for illuminating discussions. They also thank Daniel Quillen for a stimulating conversation.

\section{References}

1. Flato, M., et D. Sternheimer: Poincaré partially integrable local representations and mass-spectrum, Commun. Math. Phys. 12, 296 (1969).

2. Nelson, E.: Analytic vectors. Ann. Math. 70, 572 (1959). 
3. Jost, R.: Eine Bemerkung zu einem "Letter" von L. O'Raifeartaigh und einer Entgegnung von M. Flato und D. Sternheimer. Helv. Phys. Acta 39, 369 (1966). Segal, I. E.: An extension of a theorem of L. O'Raifeartaigh. J. Funct. Anal. 1, 1 (1967). - Sternheimer, D.: Propriétés spectrales dans les représentations de groupes de Lie, J. Math. pures et appl., 47, 289 (1968).

4. Hochschild, G.: The structure of Lie groups. San Francisco: Holden-Day 1965.

5. Nagata, M.: Local rings. New York: Interscience 1962. - Artin, M., and B. Mazur: On periodic points (Section 1). Ann. Math. 81, 82 (1965).

6. Demazure, M.: Classification des algèbres de Lie filtrées (Séminaire Bourbaki, exposé $\mathrm{n}^{\circ} 326$, Juin 1967).

\author{
M. Flato \\ D. Sternheimer \\ Institut Henri-Poincaré \\ 11, rue Pierre et Marie Curie \\ F 75 Paris $\left(5^{\mathrm{e}}\right)$
}

